Military Technical College Kobry El-Kobbah, Cairo, Egypt.

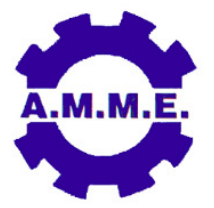

$17^{\text {th }}$ International Conference on Applied Mechanics and Mechanical Engineering.

\title{
TOPOLOGY OPTIMIZATION OF A PRESSURE CHAMBER DESIGN USING COMPUTER AIDED DESIGN SOFTWARE
}

\author{
Y. I. El-Shaer*
}

\begin{abstract}
Shape optimization is an important objective during construction and design of mechanical components. For new designs, selecting the shape and material distribution within the designed parts requires experience and/or exhaustive trials. Topology optimization enables optimum design with less iterations. In the present work, topology optimization is used to enhance an existing design of a pressure chamber to reduce the weight, while keeping the safety and performance requirements of the chamber. Solid-thinking Inspire software was used to reduce the weight of two parts, while maximizing their stiffness. Von-Mises stresses and maximum deformation were monitored as well to ensure the safety of the modified design. It was possible to reduce the overall weight of one part by $50 \%$.
\end{abstract}

\section{KEYWORDS}

Topology Optimization, Light Weight, Design, Pressure Chamber, HyperWorks.

\footnotetext{
* Egyptian Armed Forces.
} 


\section{INTRODUCTION}

Decreasing component weight while achieving same or better performance in terms of stiffness and durability is a primary design objective specially in the Aerospace industry. Reduced component weight results in lower fuel consumption and higher loading capacity. Without experience, traditional design techniques fail to achieve these goals due to time consuming nature of the design-analyze-modify cycle. New optimization techniques, such as Topology Optimization (TopOpt), provide optimal initial designs and significantly reduce the engineering time. Recent developments in this field result in easy to use software with CAD enabled outputs and robust smoothing techniques. Typical TopOpt software are ATOM [1], HyperWorks Solid thinking [2] and Ansys Shape Optimization [3]. Today, these optimization techniques are widely used in different scales of the industry (from micro scale electronics components to aircrafts and bridges parts)[4, 5]. In the present work, TopOpt was used to improve an initial design of an industrial pressure chamber. The objective was to reduce the weight of the chamber while keeping the design and safety requirements.

\section{PROBLEM DEFINITION}

The pressure chamber shown in Fig. 1 is used to inspect different sealing techniques at high pressure for steam boilers and chemical industry applications. Two parts are subjected to TopOpt, which are the chamber cover and the opening mechanism support. The applied forces for the support were calculated as the load required to lift the cover weight. While for the cover, it was the testing internal pressure.
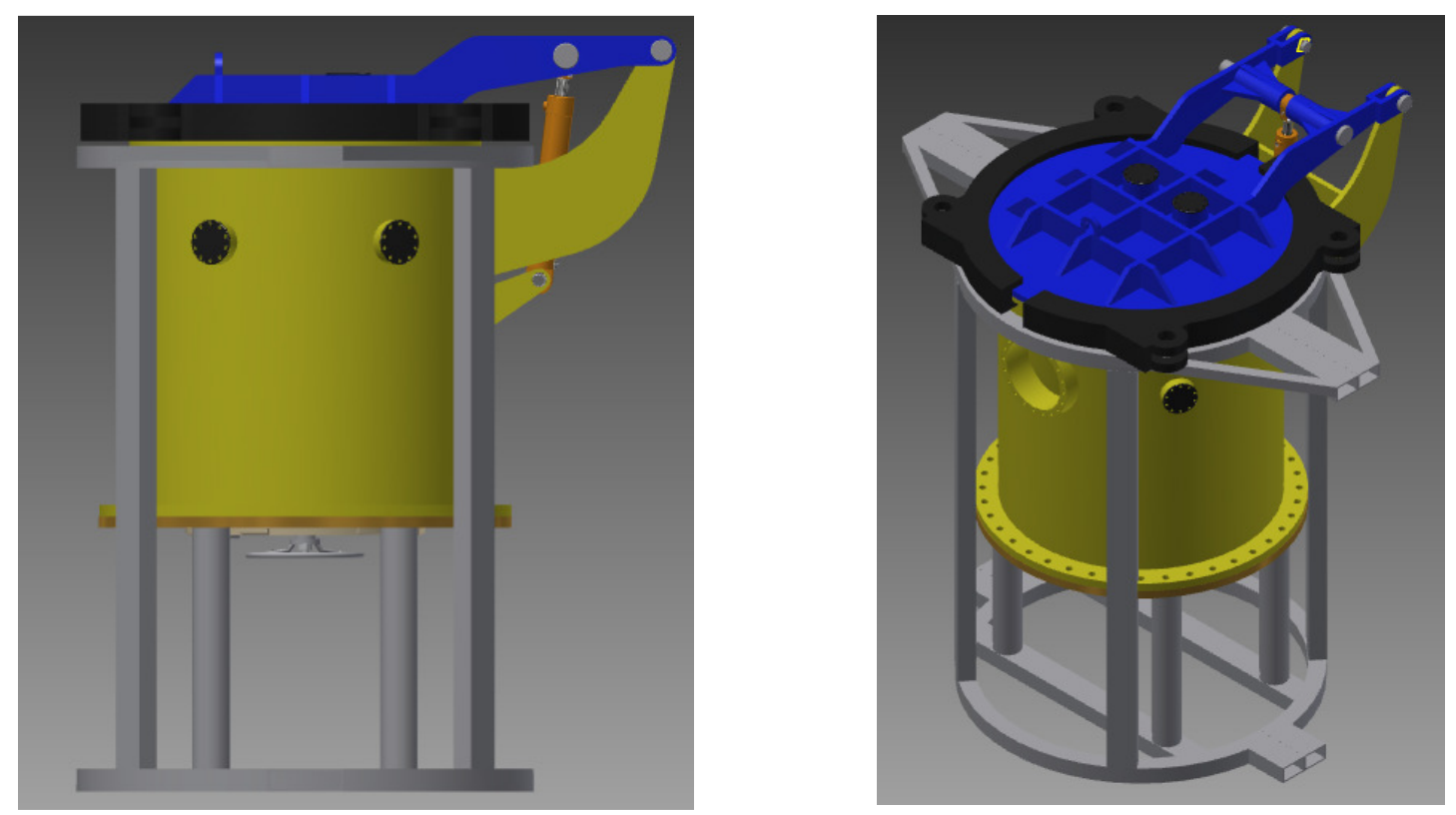

Fig. 1. Original design to be modified. 


\section{TOPOLOGY OPTIMIZATION}

The general set-up for optimal shape design can be formulated as follow. Consider the body shown in

Fig. 2 which occupies a domain $\mathbf{\Omega}^{\text {mat }}$ which is a part of larger domain $\boldsymbol{\Omega}$. The reference domain $\boldsymbol{\Omega}$ is chosen to allow for definition of applied loads $\boldsymbol{f}$ and boundry conditions $\mathbf{t}$, while $\mathbf{u}$ is the associated displacement field. The optimal design problem can be defined as the optimal design of the stiffness tensor that minimizes the compliance. Using discritization, the optimization problem can be formulated as [4, 6]:

$$
\min _{u, E_{e}} f^{T} \cdot u \quad \text { such that } K\left(E_{e}\right) \cdot u=f, E_{e} \in E_{a d}
$$

where $\boldsymbol{u}$ and $\boldsymbol{f}$ are the displacement and load vectors respectively, $\boldsymbol{K}$ is the stiffness matrix which depends on the stiffness $E_{\boldsymbol{e}}$ of each element $\mathbf{e}$ from 1 to $N$ elements, and $\boldsymbol{E}_{a d}$ is the admissible stiffness tensors for the elemnts of the design problem.

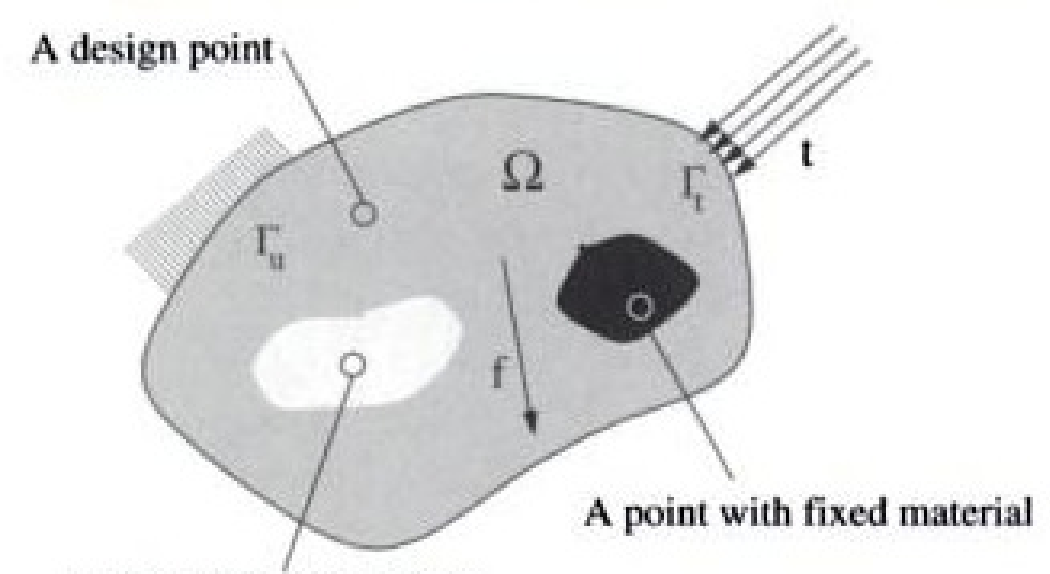

A point with no material

Fig. 2. The generalized 2-D shape design problem [4].

Finite element analysis programs are used to solve the optimization task mentioned before. In order to perform TopOpt task, the part under investigation is first modeled using available CAD software, then imported to the solver. The part is divided into model space and design space where the modifications and reduction can be done during the optimization process. Boundary conditions and loads are applied to the part. Also, any geometrical or shape constrains that are related to the expected production method can be applied as well (such as symmetry, single draw, split draw, stamping and extrusion). In this research, the objective function was to maximize the stiffness of the structure (which is equivalent to minimization of the strain energy), while reducing the volume up to specific value (up to $20 \%$ of the initial design space). 


\section{RESULTS}

\section{Topology Optimization of the Cover Mechanism Support}

First, the support was investigated for different reduction ratios using Hyperworks SolidThinking Inspire [2]. The objective was to maximize stiffness for different reduction ratios up to $20 \%$ of the initial design while monitoring the maximum VonMises stresses and displacement. The design space was the whole support except for the fixation side and the joint cylinder. The geometrical constrain was first set to Extrusion (for plasma, laser, and wire cut production), and then to a split draw (for casting and forging).

Topology optimization results are shown in Figure 3 while stress and displacement contours are shown in Figures 4 and 5, respectively.

As shown in Figure 6 a maximum reduction ratio $20 \%$ of the initial design (Reduction ratio=Optimized design volume/Original design volume) leads to an increase in stresses and displacement, but still within the accepted range of typical structural steels used in the market. If the reduction is limited to $50 \%$ of the initial design space a minor increase is observed in stress and displacement.

Adding split draw geometrical constrain, so that the product can be manufactured using casting or forged process, results in further decrease of the stresses and displacement compared to the corresponding part with extrusion constrain (Figures 7 and 8).

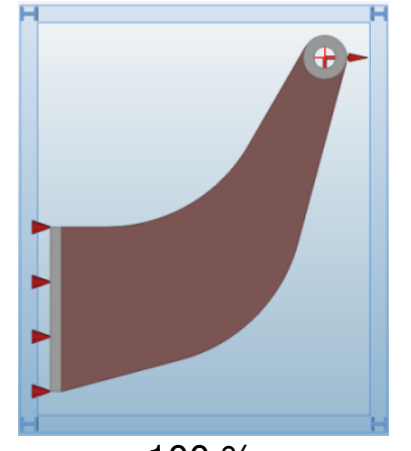

$100 \%$

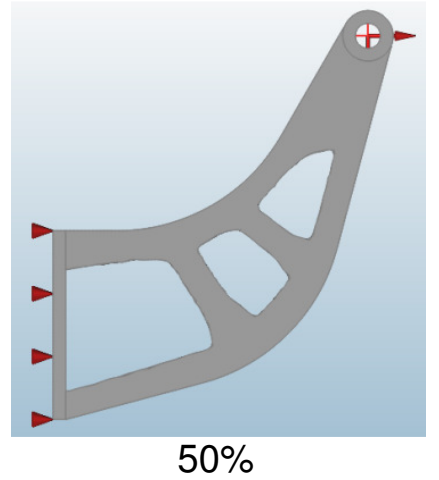

$50 \%$

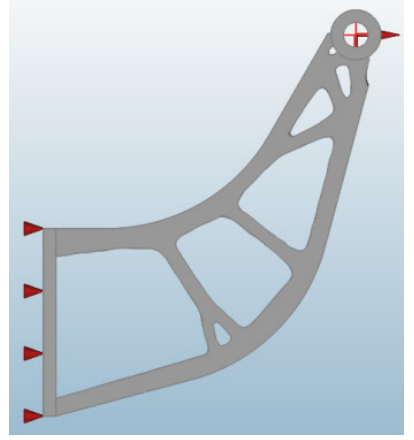

$40 \%$

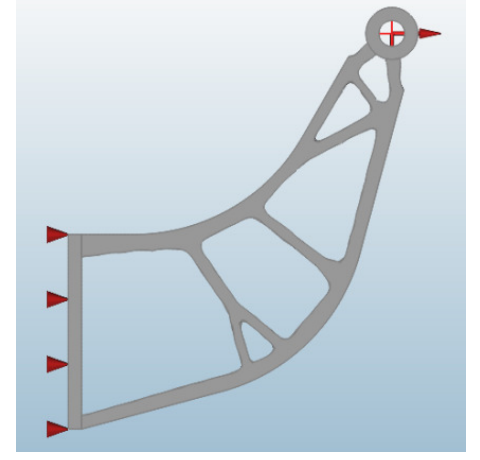

$30 \%$

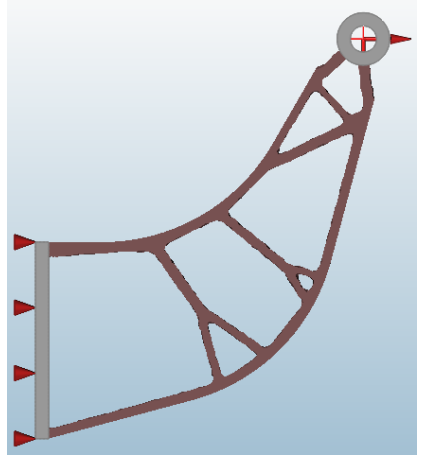

$20 \%$

Figure 3. Support Topology Optimization results for different reduction ratios. 


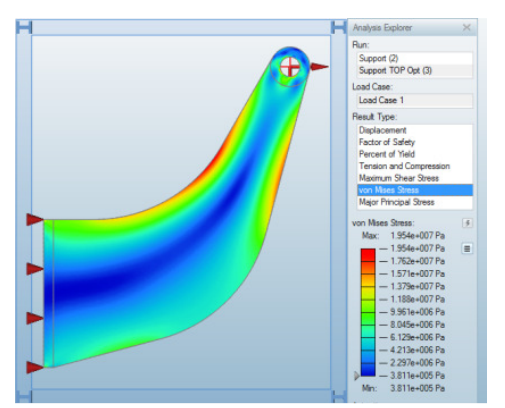

$100 \%$

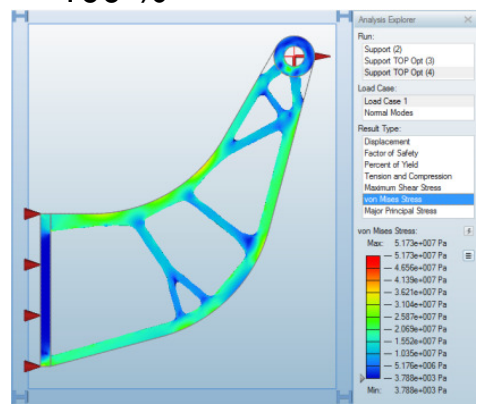

$30 \%$

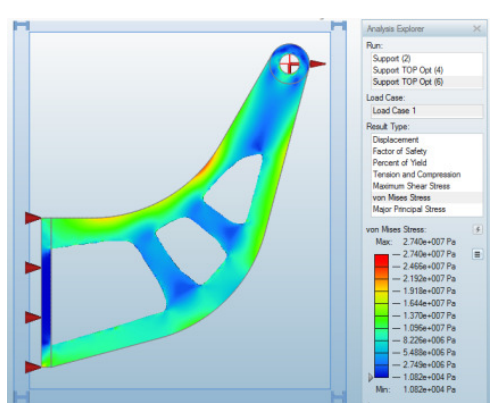

$50 \%$

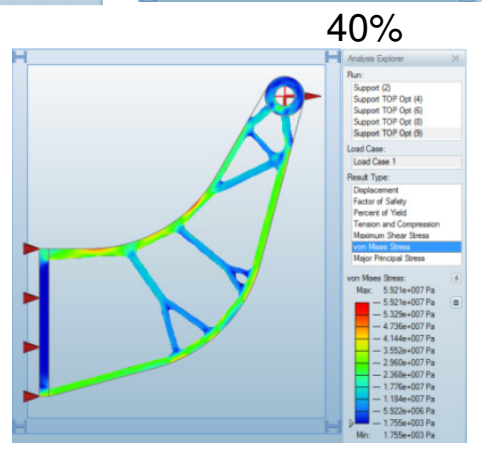

$20 \%$

Fig. 4. Support Von-Mises stresses contours for different reduction ratios.

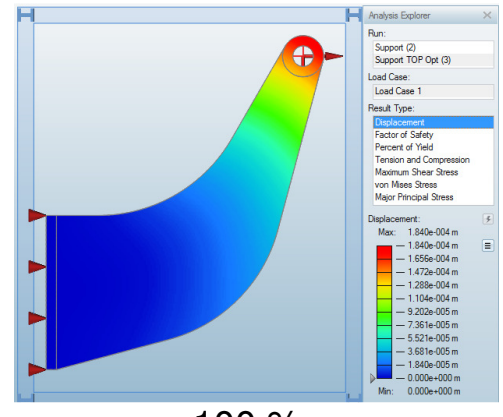

$100 \%$

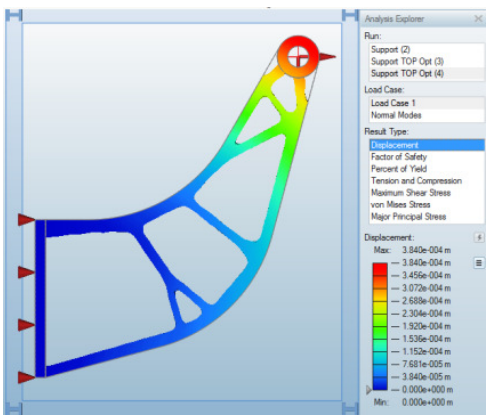

$30 \%$

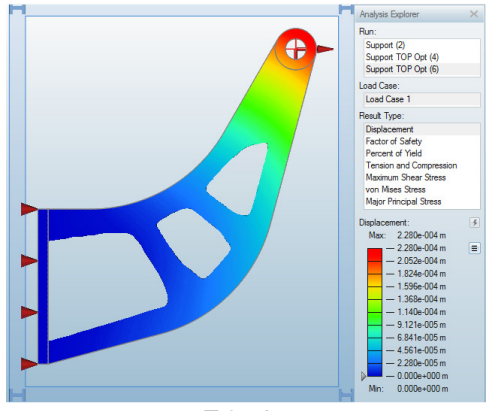

$50 \%$

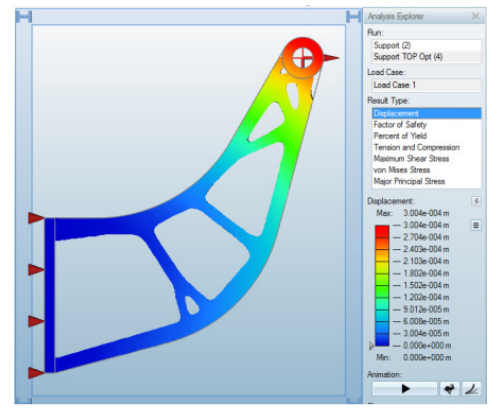

$40 \%$

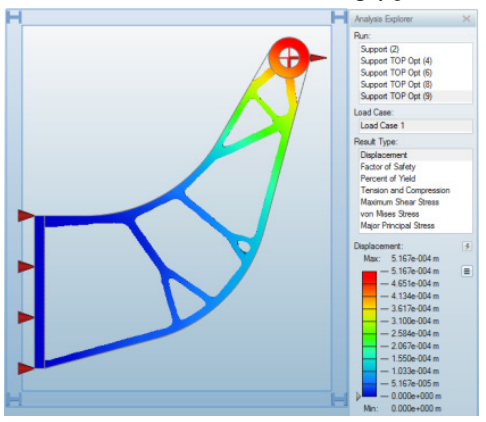

$20 \%$

Fig. 5. Support Displacement contours for different reduction ratios. 


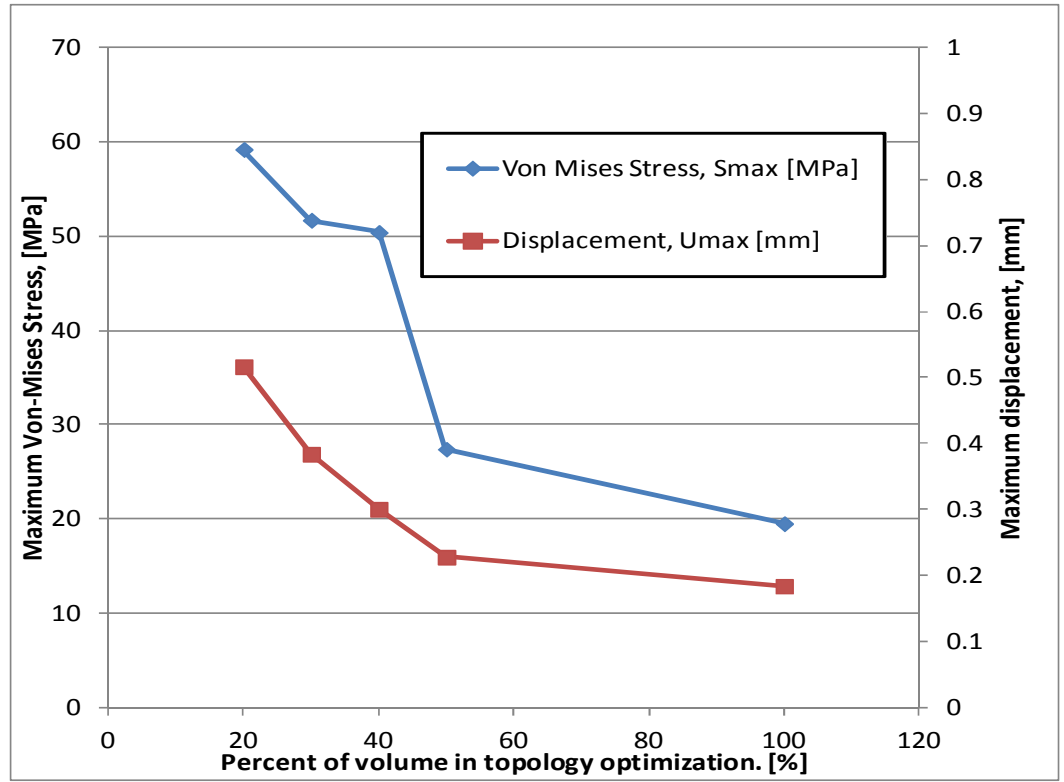

Fig. 6. Maximum support displacement and stresses for different reduction ratios.

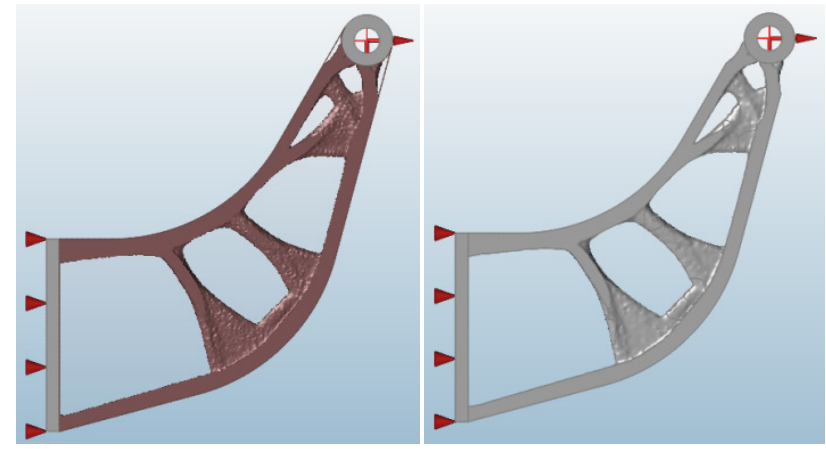

Fig. 7. Changing shape control from extrusion to split draw (30\% reduction ratio).

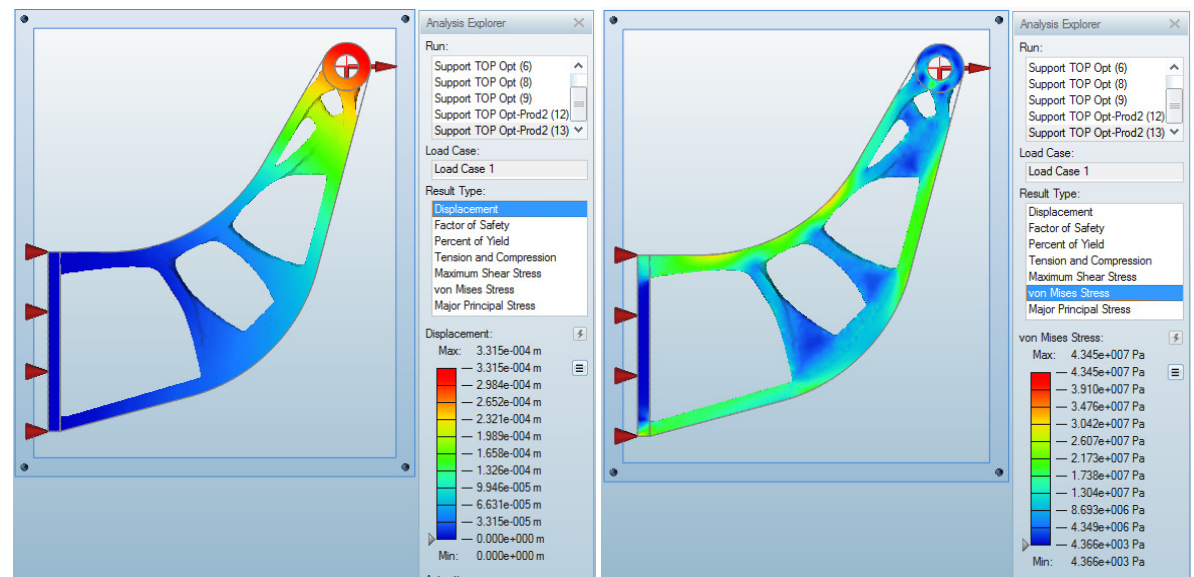

Fig. 8. Stresses and displacement for split draw. 


\section{Topology optimization of the Pressure Chamber Cover}

The pressure chamber cover was studied using the same procedure explained earlier. The cover consists of the bottom cylindrical plate, which cannot be modified due to function requirement, and the upper stiffeners which was investigated to obtain suitable stiffener shape and orientation for different geometrical constrains.

Extrusion was used first as the geometrical constrain. Different topology optimization results are shown in Figures 10,11 and 12. The maximum stresses and displacement are shown in Figure 13. Another geometrical constrains was applied (symmetrical and single draw) and the obtained results are shown in Figures 14, 15 and 16.

Due to the complicated shapes obtained and to get a feasible topology, a proposed shape was introduced and parametrically optimized to fulfill the design requirements. The proposed shape was built in accordance with the topology optimization results obtained and is shown in Figure 17.

Four parameters were selected for optimization, Rim Diameter, Thickness, Rib Length and chamfer distance. Using the proposed design, it was possible to obtain lower stresses, displacement while the mass reduction was not significant (Figure 18).

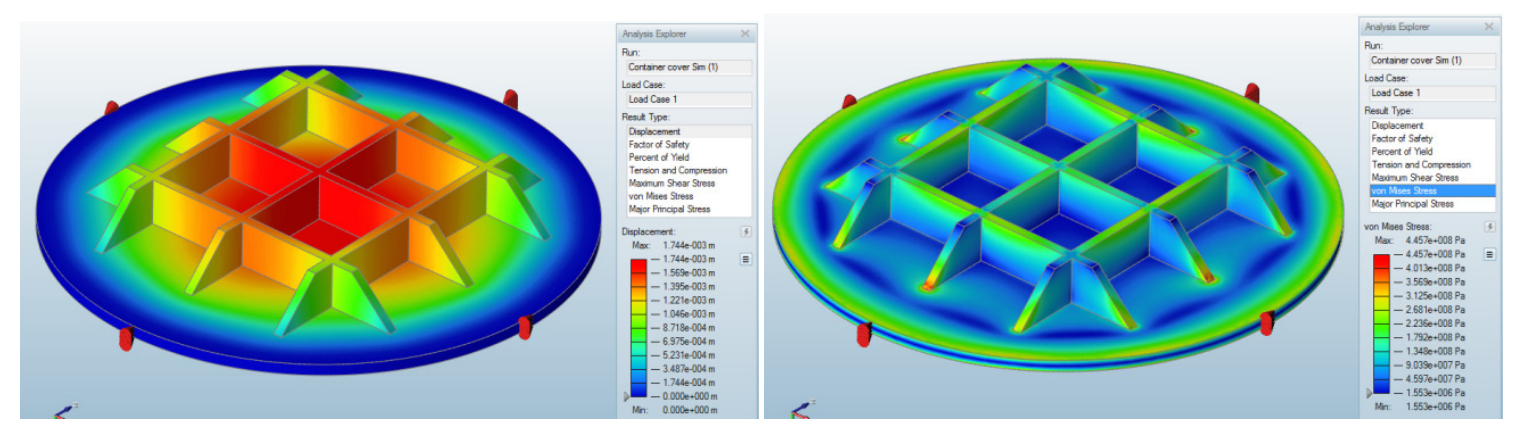

Fig. 9. Stresses and displacement contours for original cover design.

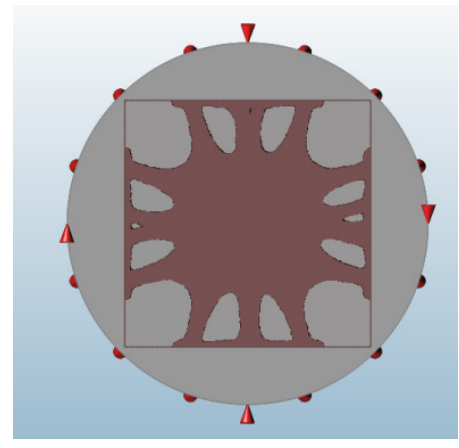

$50 \%$ Extrusion

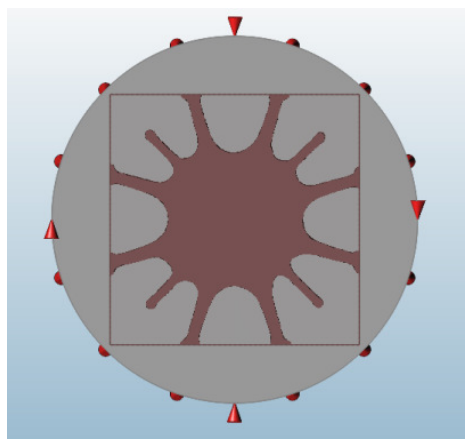

$30 \%$ Extrusion

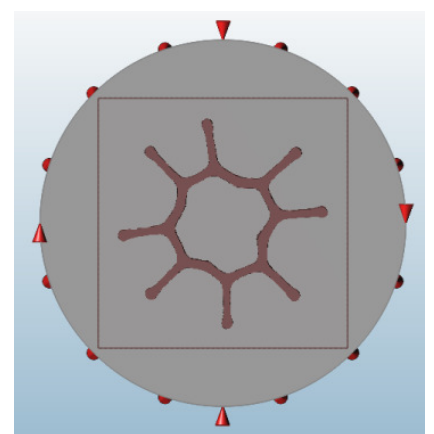

$15 \%$ Extrusion

Fig. 10. Topology optimization of the chamber cover. 


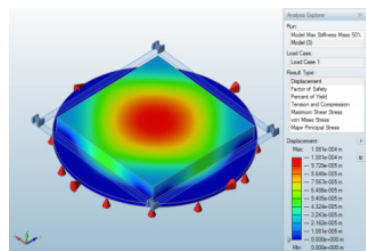

$100 \%$

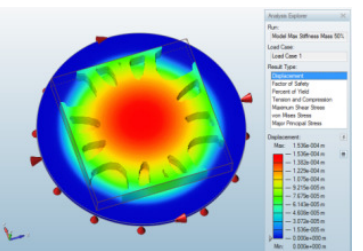

$50 \%$

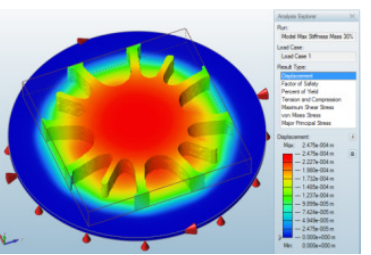

$30 \%$

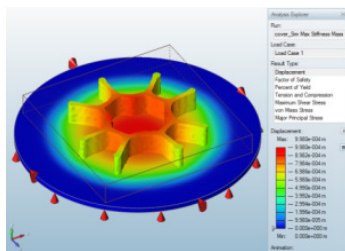

$15 \%$

Fig. 11. Cover displacement contours for different reduction ratios.
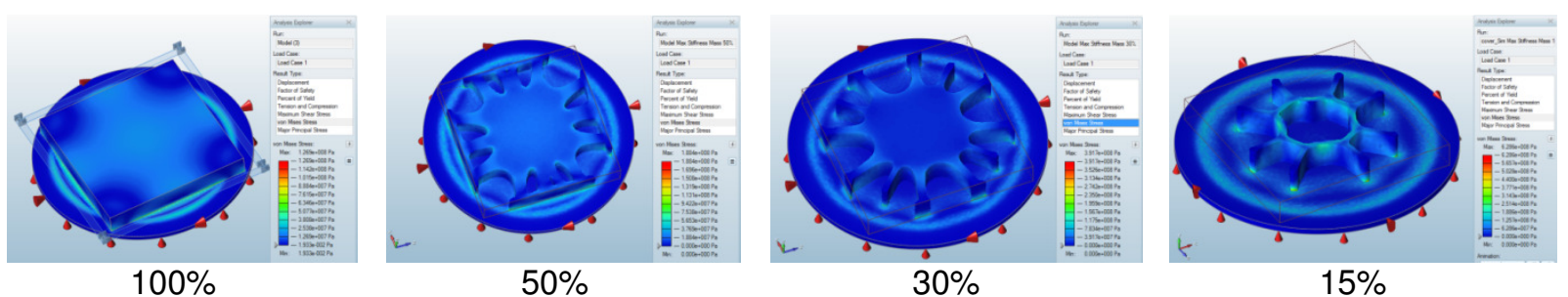

Fig. 12. Cover Von-Mises stresses contours for different reduction ratios.

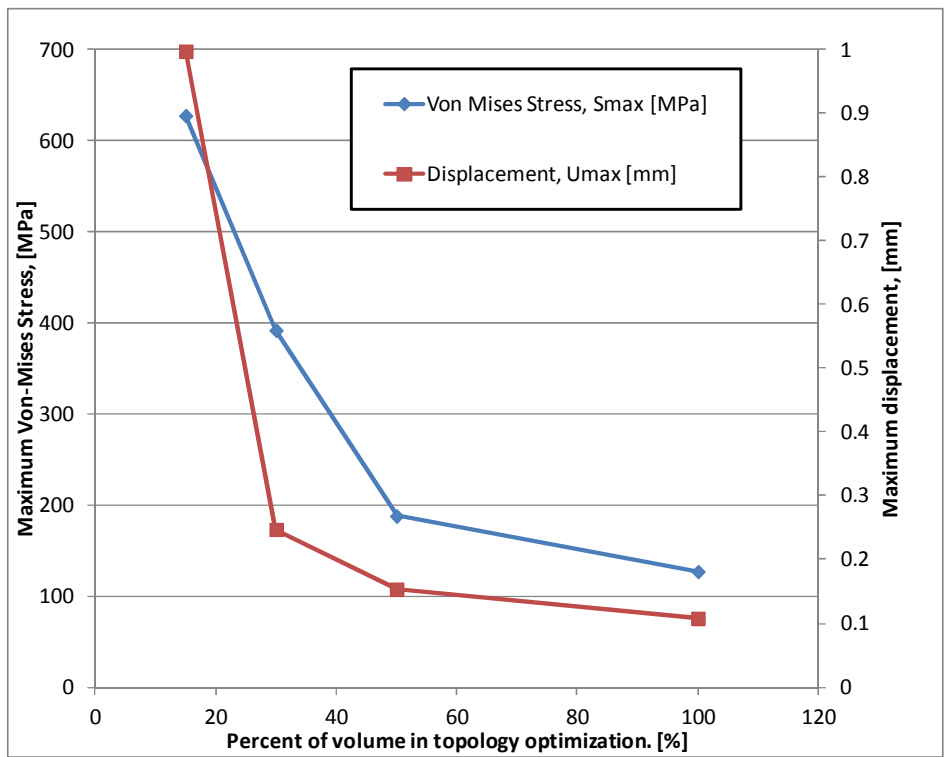

Fig. 13. Maximum cover displacement and stresses for different reduction ratios.
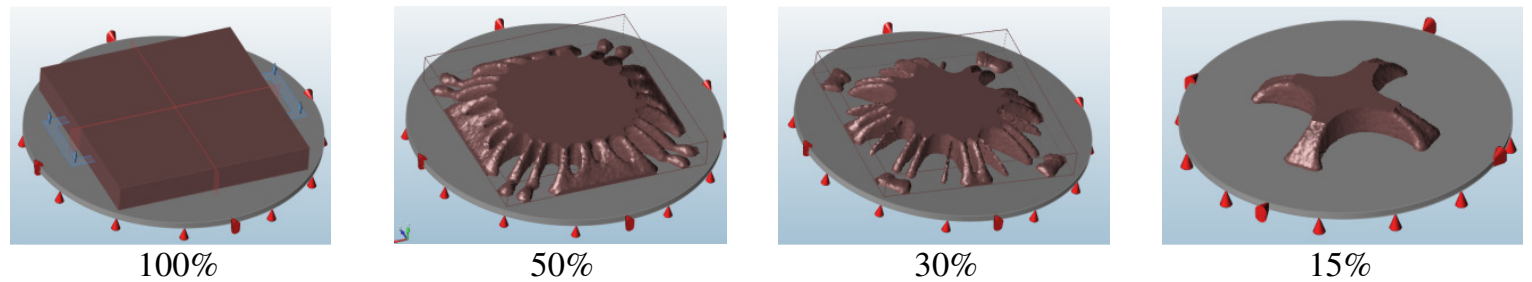

Fig. 14. Topology Optimization results obtained when adding Symmetry and single draw to the design space. 


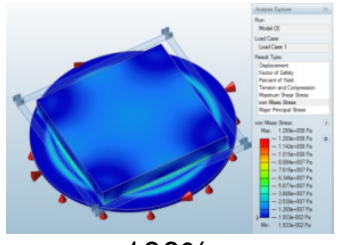

$100 \%$

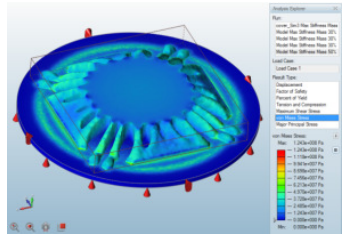

$50 \%$

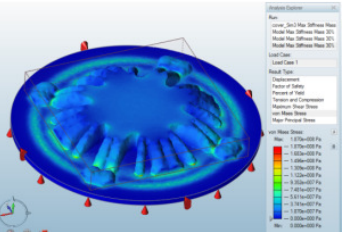

$30 \%$

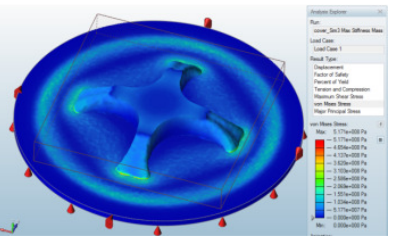

$15 \%$

Figure 15. Stress contours obtained when adding Symmetry and single draw to the cover design space.

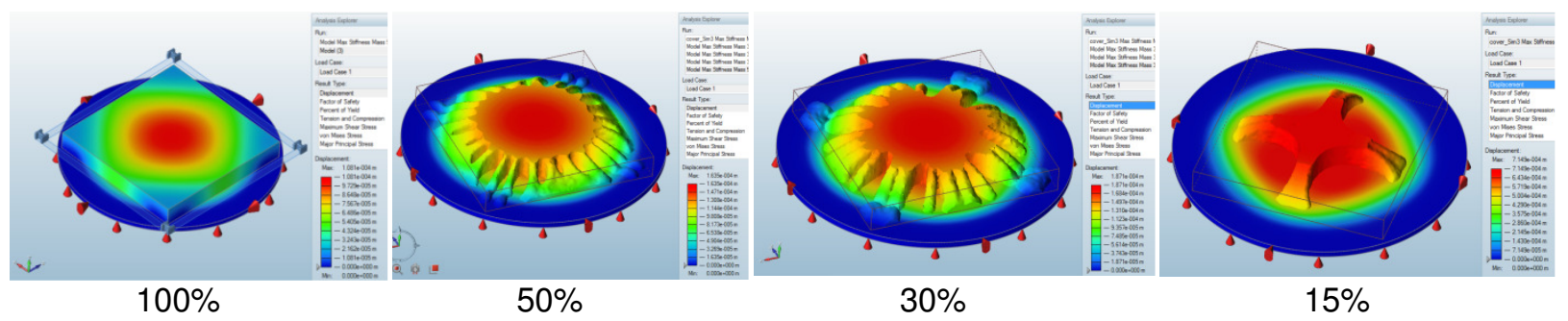

Figure 16. Displacement contours obtained when adding Symmetry and single draw to the cover design space.

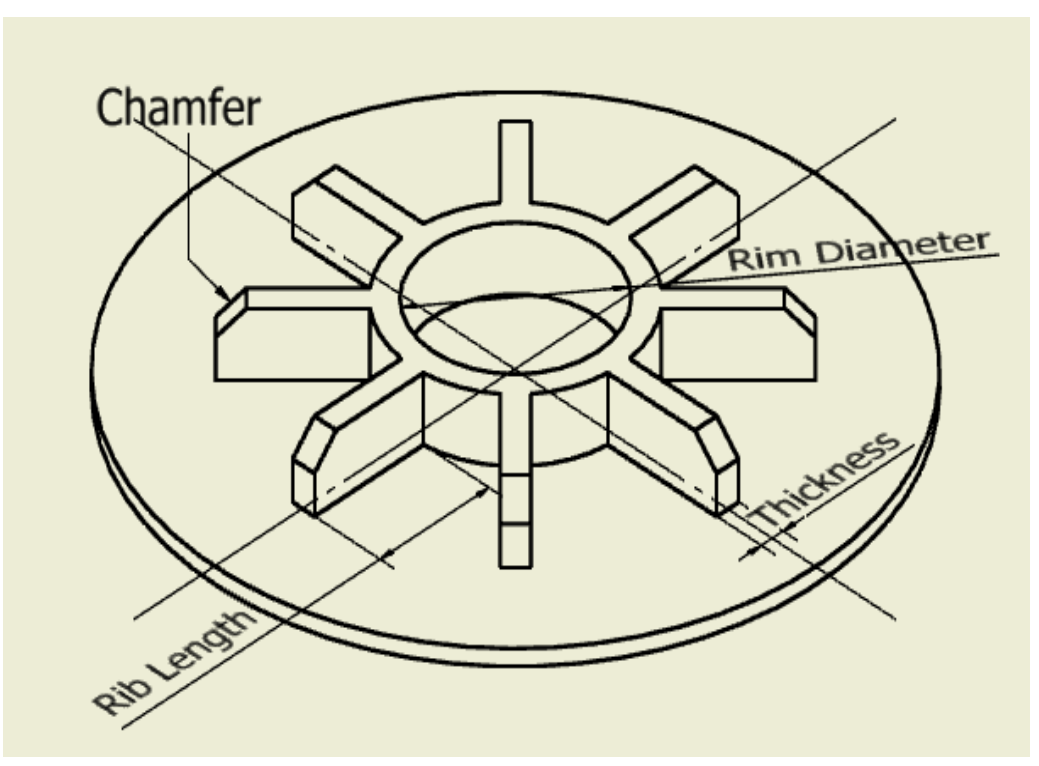

Figure 17. Proposed cover design based on the topology optimization results obtained. 

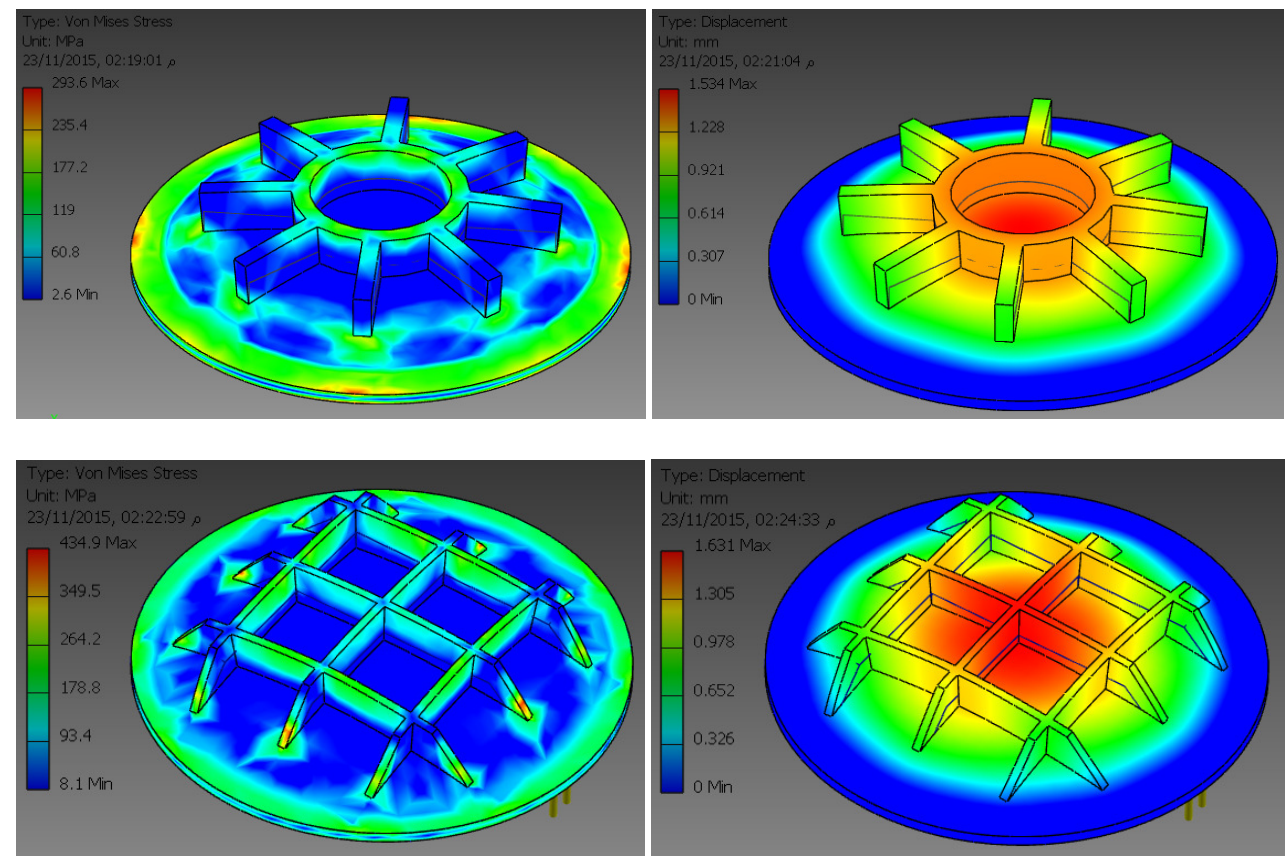

Figure 18. Comparison between the original design and that obtained using topology then parametric optimization techniques.

\section{CONCLUSIONS}

Using topology optimization techniques, it was possible to get a mass reduction up to $50 \%$ for the Support under investigation with an acceptable change in the stiffness or stress, which does not affect the performance or safety measures of the component. For the pressure vessel cover, the topologies of the optimized geometry did not give feasible shape from manufacturing point of view. As a result, topology optimization results were used as baseline for further design iterations or parametric optimization. The enhancements to the original design were not significant (which indicates a previously optimized configuration). Using this technique is highly beneficial for the design of new parts with no previous experience, and for bulky parts. However, depending on the part geometry, using hybrid design algorithm (utilizing topology optimization and parametric optimization) is needed to obtain significant enhancement. It is also important to account for excessive manufacturing cost that should be included in the final design stage to justify using the proposed configuration of parts.

\section{REFERENCES}

[1] Becene, A.T., Topology Optimization of Nacelle Components with ATOM, in SIMULIA Customer Conference. 2012.

[2] Altair. SolidThinking Inspire. 2015; Available from: http://www.solidthinking.com/. 
[3] Ansys Workbench. 2015, Ansya Inc.

[4] Martin Philip Bendsoe, O.S., Topology Optimization: Theory, Methods, and Applications. 2003: Springer.

[5] Topology Optimization Research Group. 2015; Available from: http://www.topopt.dtu.dk/.

[6] Johnsen, S., Structural Topology Optimization: Basic Theory, Methods and Applications, in Department of Engineering Design and Materials. 2013, Norwegian University of Science and Technology. 